\title{
Post-Traumatic Septic Bone Sequestration in Three Dogs
}

\author{
Graham Hayes $^{1}$ Karen Harris ${ }^{2}$ Sorrel Langley-Hobbs ${ }^{3}$ \\ ${ }^{1}$ Kentdale Veterinary Orthopaedics, Unit 2B Moss End Business \\ Village, Crooklands, Cumbria, United Kingdom \\ 2 Southern Counties Veterinary Specialists, Unit 6 Forest Corner Farm, \\ Hangersley, Ringwood, Hampshire, United Kingdom

\begin{abstract}
Address for correspondence Graham Hayes, MA VetMB CertSAS DipECVS, Kentdale Veterinary Orthopaedics, Unit 2B Moss End Business Village, Crooklands, Cumbria LA7 7NU, United Kingdom
\end{abstract} \\ (e-mail: gmh327@gmail.com).
}

${ }^{3}$ Langford Veterinary Services, University of Bristol, Langford, Bristol, United Kingdom

VCOT Open 2019;2:e9-e15.

\begin{abstract} Keywords

- osteomyelitis

- bone diseases

- infections

Formation of discreet bony sequestra is reported in three dogs presenting with posttraumatic osteomyelitis with no previous history of orthopaedic surgery. Two cases had a presumed or confirmed history of dog bites, while the third case had a fracture treated conservatively. All three were treated with surgical debridement and sequestrectomy with good outcome.
\end{abstract}

\section{Introduction}

Sequestra formation normally requires devitalized bone and an infectious agent. Opportunistic infectious agents may colonize a devitalized bone fragment or may be primary pathogens, which cause osteonecrosis during colonization. Both scenarios may lead to bone sequestration. Bone sequestration most commonly occurs as a complication of orthopaedic surgery but may also arise following trauma or bacteraemia. ${ }^{1,2}$ Bone sequestration is a relatively common presentation in horses and cattle but is unusual in dogs and cats. $^{3-5}$ Three cases of bone sequestration are reported that are unusual in that they developed in dogs with no previous history of orthopaedic surgery.

\section{Case One}

A 5-month-old female entire Dalmatian initially presented to the referring veterinary surgeon with an acute onset, nonweight bearing lameness of the left hindlimb. The puppy was kept with its sibling and there was no history of trauma reported. No palpable or visible abnormalities were found on clinical examination of the limb but the puppy was pyrexic $\left(39.8^{\circ} \mathrm{C}\right.$ ) so carprofen (Rimadyl; Zoetis, $2 \mathrm{mg} / \mathrm{kg}$ per os [PO] $\mathrm{q} 12 \mathrm{~h}$ ) was prescribed. Four days later there was cellulitis around the metatarsus and the popliteal lymph node was enlarged. The puppy was started on amoxicillin-clavulanate (Noroclav; Norbrook Laboratories, $15 \mathrm{mg} / \mathrm{kg}$ q12h) but the swelling progressed. Despite changing the antibiotic medication to clindamycin (Antirobe; Zoetis $9 \mathrm{mg} / \mathrm{kg}$ PO q12h), neither the lameness nor the swelling improved over the following 2 weeks.

At the time of referral, 3 weeks following the onset of lameness, the dog was moderately (3/5) lame on the left pelvic limb and there was moderate enlargement of the inguinal and popliteal lymph nodes. The left hind foot was warm, with cellulitis extending from the distal tarsus to the digits. At that stage the puppy was normothermic $\left(38.4^{\circ} \mathrm{C}\right)$. The remainder of the clinical examination was considered normal.

Routine biochemistry and haematology were unremarkable, with no evidence of systemic inflammation. Radiography of the metatarsus showed a bone-within-bone image in the second and fourth metatarsal bones ${ }^{6}$ (-Fig. 1A). Both the second and fourth metatarsals were enlarged with medullary sequestra and diffuse periosteal new bone, consistent with involucra formation. A cloaca was present in both the medial cortex of the second metatarsal and the dorsal aspect of the fourth metatarsal bone. There was soft-tissue swelling around the metatarsus, particularly medially and dorsally. Ultrasound examination was performed, confirming the presence of a cortical defect in the second and fourth metatarsals with a small amount of fluid accumulation in the soft-tissues received

January 30, 2019

accepted after revision

May 16, 2019
DOI https://doi.org/

10.1055/s-0039-1692974. ISSN 2625-2325.
๑) 2019 Georg Thieme Verlag KG
Stuttgart · New York

License terms

(1)(1) 


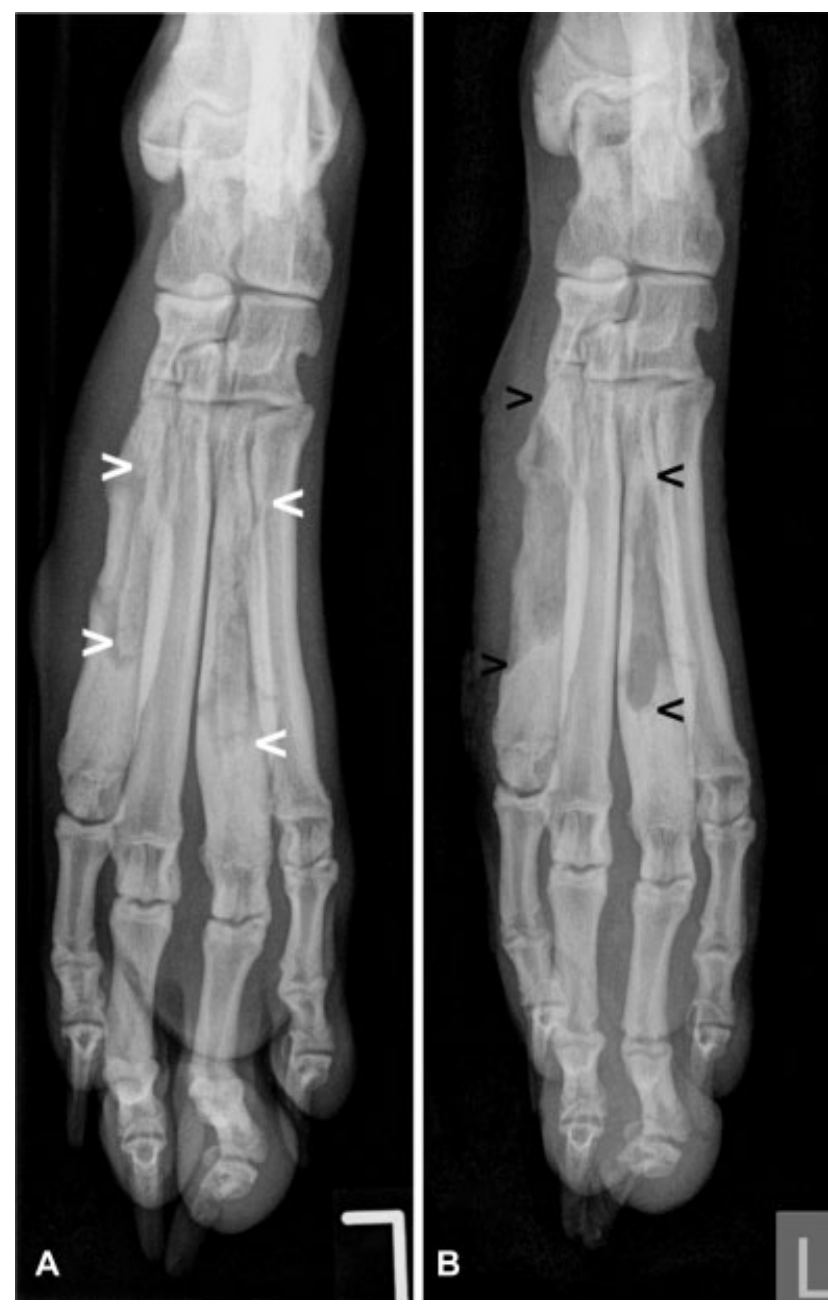

Fig. 1 Dorsoplantar radiographs of case 1. (A) Preoperative view showing bone fragments within the medullary cavity of the second and fourth metatarsal bones (arrowheads). On the medial aspect of the second metatarsal is a focal cortical defect visible, consistent with cloaca formation. (B) Immediate postoperative view, confirming complete sequestrectomy.

medially and dorsally. Aspirates from the popliteal lymph node were consistent with reactive hyperplasia, and a diagnosis of osteomyelitis with sequestration was made.

Surgical debridement of the lesions was performed the following day under general anaesthesia. Surgery was facilitated using an Esmarch's tourniquet providing a nearly bloodless surgical field. A skin incision was made over the length of the medial aspect of the second metatarsal bone. The cloaca was visible as a small hole in the medial cortex of the bone with purulent material exuding from it. A highspeed burr was used to make a longitudinal slot in the bone to allow removal of the sequestrum from the medullary cavity. The sequestrum was a partial circumference cortical fragment. The medullary cavity of the bone was gently curetted, removing remaining necrotic soft-tissue and then copiously lavaged with sterile $0.9 \%$ saline solution, followed by routine closure of the soft-tissues. A separate incision was made over the dorsal aspect of the fourth metatarsal bone and the sequestrum removed in a similar manner. Both sequestra were of similar shape and size. Following seques- trectomy, the patient was treated perioperatively with amoxicillin-clavulanate (Augmentin; GlaxoSmithKline UK Ltd., $20 \mathrm{mg} / \mathrm{kg}$ intravenously). Postoperative radiographs (-Fig. 1B) demonstrated completeness of sequestrectomy and the limb was bandaged postoperatively. Opiate analgesia was given overnight and the dog was discharged the following day with carprofen (Rimadyl; Zoetis, $2 \mathrm{mg} / \mathrm{kg}$ PO q12h for 7 days) for regular dressing changes at the referring veterinarian. No postoperative antibiotic medications were prescribed as the surgical wounds were regularly inspected during dressing changes and resolution of inflammation and lameness could be monitored.

A sparse growth of Pasteurella pneumotropica was cultured from the sequestrum from the second metatarsal, resistant to metronidazole and clindamycin but sensitive to all other antibiotic medications tested. Blood culture testing was not performed.

Subsequent recovery was uneventful, with the dog reportedly fully weight bearing on the operated limb within a few days of discharge. The surgical incisions healed normally and radiographs 18 days after surgery showed smooth periosteal callus formation and infilling of the bony slots. The dressing was removed at this point and the dog was placed on a progressive plane of lead exercise for 4 weeks. After 4 weeks, the dog was reported to have made a full recovery and was sound on the limb. No further problems were reported over the following 2 years.

\section{Case Two}

A 3-year-old male neutered Springer Spaniel was presented to the referring veterinary surgeon with discharging sinuses over the proximomedial aspect of the left tibia. The dog was owned by a rescue centre and was known to have sustained a fracture of the left tibia at 9 weeks of age. Although fracture repair or amputation had been recommended by a veterinary surgeon at the time, neither were performed and the dog had eventually come into the possession of the rescue centre. Radiographs performed by the referring veterinary surgeon were suggestive of chronic osteomyelitis with sequestrum formation. Despite 8 weeks of cephalexin (Rilexine; Virbac, $25 \mathrm{mg} / \mathrm{kg}$ PO q12h), the sinuses continued to discharge serosanguinous fluid and the dog was referred.

At the time of referral, the dog was not lame and there was minimal pain on palpation of the left hindlimb. Three sinuses were present on the skin adjacent to the medial tibia (-Fig. 2A) and there was local lymphadenopathy. The dog was normothermic $\left(38.2^{\circ} \mathrm{C}\right)$ and the remainder of the clinical examination was considered normal.

Routine biochemistry and haematology were also unremarkable, with no evidence of systemic inflammation. Radiographs of the left tibia revealed a healed complex tibial fracture with a segmental sequestrum of the tibial diaphysis (-Fig. 3A, B). An involucrum was present in the proximal tibia and the tibial defect was bridged by a hypertrophic fibula which had formed a synostosis with the tibia distal to the lesion. The left tibia was $10 \mathrm{~mm}$ shorter than the right tibia. 


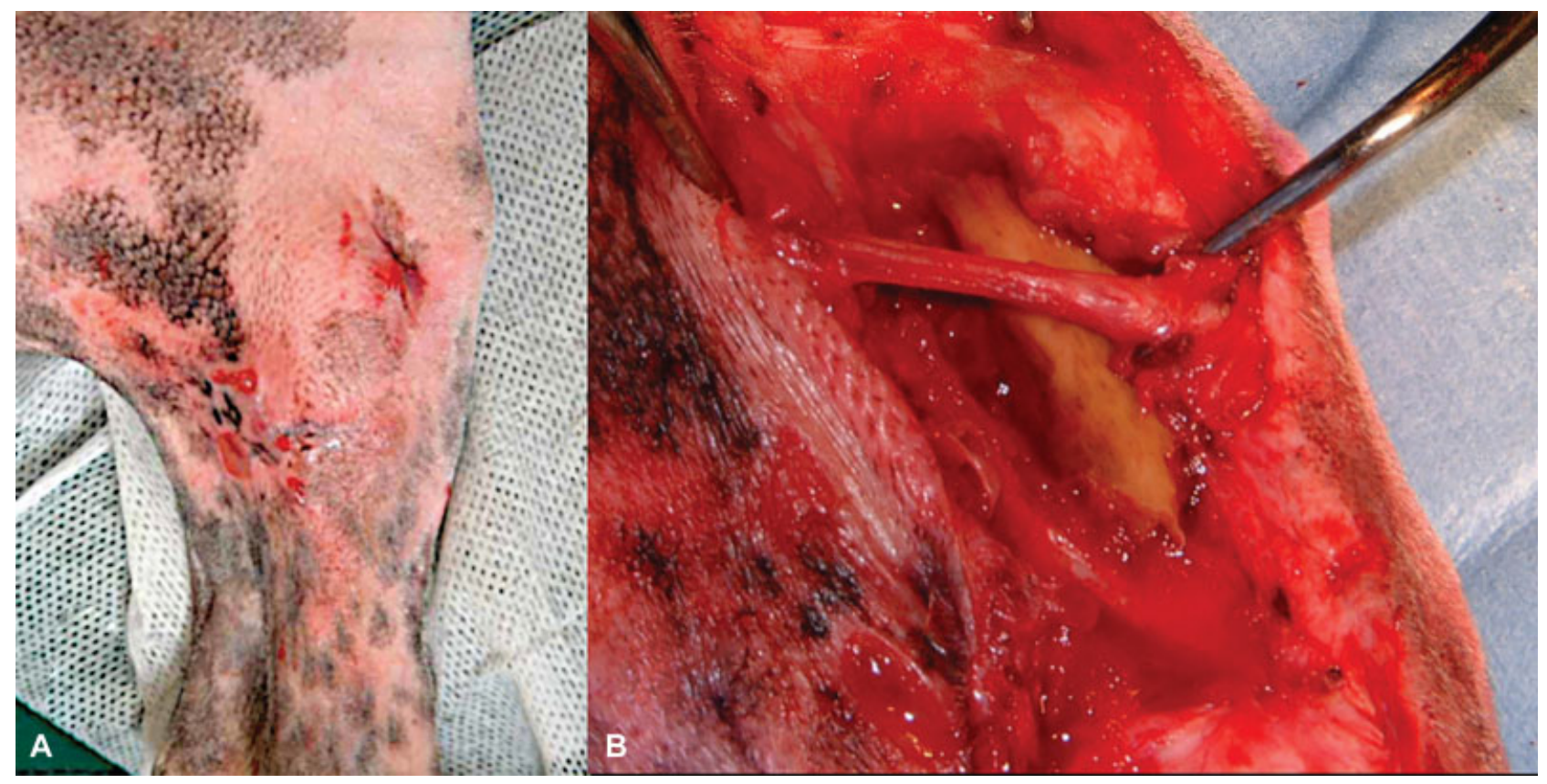

Fig. 2 Pre- (A) and intraoperative (B) views of case 2. (A) Multiple draining sinuses on the medial aspect of the tibia. (B) The sequestrum in situ devoid of soft-tissue attachments.
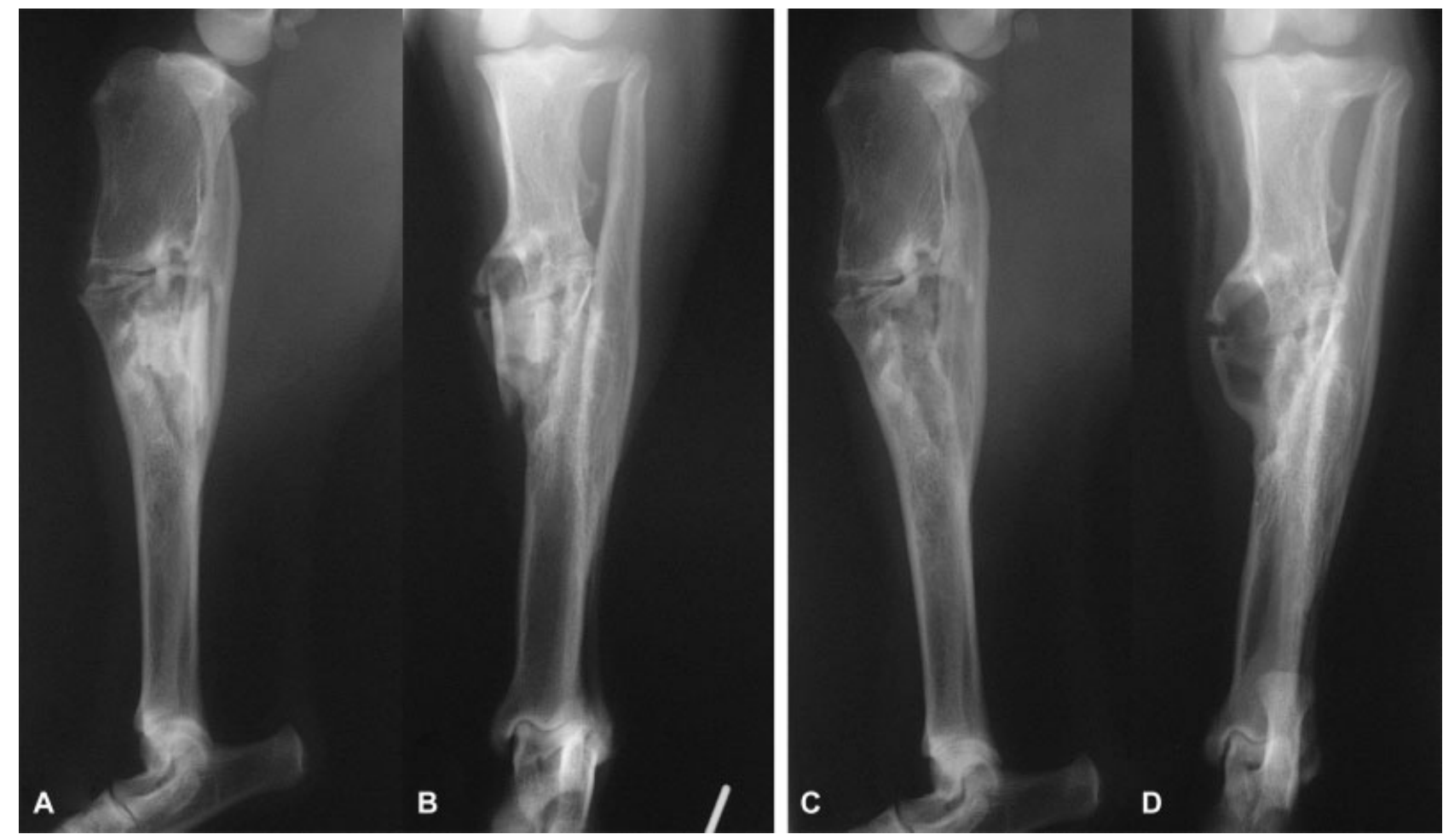

Fig. 3 Mediolateral (A, C) and caudocranial (B, D) radiographs of case 2. (A, B) Preoperative images showing a sequestrum surrounded by an involucrum in the proximal third of the tibial diaphysis. Hypertrophy of the proximal fibula and tibiofibular synostosis. (C, D) Immediate postoperative images confirming complete sequestrectomy.

Surgical debridement of the sequestrum was subsequently performed under general anaesthesia. A skin incision was made over the medial tibia between the discharging sinuses. The sequestrum lay directly underneath the skin but had no attachments to soft-tissues or surrounding bone (-Fig. 2B). The sequestrum was greenish-yellow in colour and appeared to be a full-circumferential segment of the tibial diaphysis $\sim 25 \mathrm{~mm}$ long $\times 10 \mathrm{~mm}$ in diameter. A bacterial swab was taken for culture before intravenous amoxicillin-clavulanate (Augmentin; GlaxoSmithKline UK Ltd., 20 $\mathrm{mg} / \mathrm{kg}$ ) was administered. The surrounding cavity was curetted, flushed with sterile $0.9 \%$ saline solution and packed 
with a cancellous autograft harvested from the contralateral proximal humerus prior to routine closure of the soft-tissues. Postoperative radiographs confirmed complete sequestrectomy (-Fig. 3C, D) and the limb was bandaged. Opiate analgesia was given postoperatively and the dog was discharged the next day with carprofen (Rimadyl; Zoetis, $2 \mathrm{mg} /$ $\mathrm{kg}$ PO q12h for 6 days) and amoxicillin-clavulanate (Synulox; Pfizer, $20 \mathrm{mg} / \mathrm{kg}$ PO q12h for 6 days).

Culture of the swab identified a mixed growth of Staphylococcus sp., predominantly a $\beta$-haemolytic, coagulase negative strain but also a non-haemolytic, coagulase negative strain. Both were susceptible to a variety of antibiotic medications, including cephalexin and amoxicillin-clavulanate. Blood culture was not performed.

The dog was not presented for a scheduled re-examination 6 weeks postoperatively, but was intermittently seen by the referring veterinary surgeon who reported resolution of the discharging sinuses and only intermittent mild lameness after exercise. The dog was lost to follow-up after 6 months.

\section{Case Three}

A 3-year-old female neutered working Labrador Retriever presented to the referring veterinary surgeon with a 3-week history of progressive right forelimb lameness that was unresponsive to firocoxib (Previcox; Boehringer Ingelheim Animal Health, $5 \mathrm{mg} / \mathrm{kg}$ PO q24h). Reportedly, the dog had been bitten on the limb by another dog several weeks earlier, and the wound had healed without treatment. Radiography performed by the referring veterinary surgeon showed periosteal new bone formation in the region of the medial epicondyle of the humerus ( - Fig. 4A, B). Routine haematology and biochemistry at that time were normal, with no evidence of systemic inflammation. At referral, the dog was moderately (2/5) lame on the right forelimb and there was pain on palpation around the humeral condyle and on flexion of the elbow. The dog was normothermic $\left(38.4^{\circ} \mathrm{C}\right)$ and the remainder of the clinical examination was considered normal.
Computed tomography showed evidence of an irregular periosteal and endosteal reaction cranial and proximal to the medial epicondyle of the right humerus (-Fig. 4C). The reactive periosteal new bone formed a 'nest-like' involucrum surrounding a small sclerotic bone fragment, consistent with chronic osteomyelitis and sequestrum formation. The hair was clipped from the elbow, but there was no evidence of a draining sinus or fluid accumulation at the site of osteomyelitis and no palpable lymphadenopathy.

Surgical debridement of the lesion was performed under general anaesthesia. A medial approach was made to the right distal humerus. There was no soft-tissue sinus but incision through the periosteum revealed a small amount of exudate. The cortical involucrum was exposed and the cloaca opened with rongeurs to allow removal of the sequestrum which measured $6 \times 2 \times 3 \mathrm{~mm}$. The abscess cavity was curetted and then copiously lavaged with sterile $0.9 \%$ saline solution prior to routine closure of the softtissues. Following sequestrectomy, the patient was started on amoxicillin-clavulanate (Augmentin; GlaxoSmithKline UK Ltd, $20 \mathrm{mg} / \mathrm{kg}$ intravenously) and at discharge meloxicam (Metacam; Boehringer Ingelheim Animal Health, $0.1 \mathrm{mg} / \mathrm{kg}$ PO q24h) was added for 2 weeks.

Culture of the sequestrum identified a pure growth of Pasteurella sp., susceptible to a variety of antibiotic medications, including cephalexin and amoxicillin-clavulanate. The dog was maintained on amoxicillin-clavulanate until re-examination at 6 weeks to ensure resolution of the osteomyelitis. The re-examination revealed complete resolution of lameness, reportedly within days of surgery and uncomplicated wound healing. There was no pain on firm palpation of the distal humerus and radiography revealed resolution of the aggressive bony lesion and smooth remodelling of the cortex (-Fig. 4D, E). At this time, antibiotic medications were stopped and light exercise was resumed with unrestricted work allowed from 10 weeks postoperatively. No further problems were reported over the following 2 years.
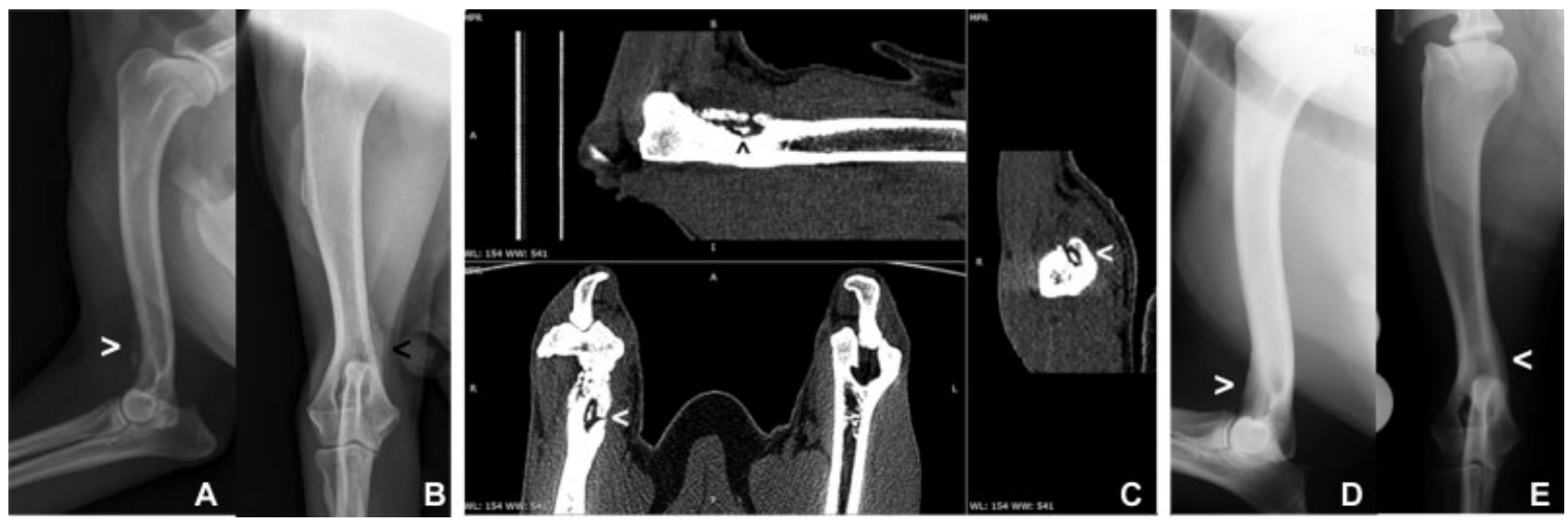

Fig. 4 Case 3: (A) Mediolateral and (B) craniocaudal preoperative radiographs of the right humerus showing irregular palisading periosteal reaction on the craniomedial aspect (arrowheads) of the distal humerus. (C) Multiplanar computed tomography reconstructions (three orthogonal views) of the distal humerus showing a bony sequestrum partially surrounded by involucrum (arrowheads). The draining cloaca is also visible. (D) Mediolateral and (E) craniocaudal radiographs of the right humerus taken 6 weeks after sequestrectomy showing smooth homogeneous periosteal remodelling of the distal humerus (arrowheads) consistent with osteomyelitis resolution. 


\section{Discussion}

A sequestrum is an avascular bone fragment and is nearly always associated with septic osteomyelitis. Sequestrum formation in dogs is almost invariably reported as a complication of fracture repair surgery, although two cases of sterile sequestration were reported in dogs with osteosarcoma. ${ }^{7,8}$ Apart from one immature dog that developed a sequestrum after a bite wound on the skull, ${ }^{9}$ the three canine cases reported here are unique in having a sequestrum developed after conservative treatment of traumatic injuries.

Normal bone and soft-tissues are relatively resistant to infection; however, a sufficient inoculum of virulent bacteria may establish an infection ( $>10^{5} \mathrm{cfu} / \mathrm{gm}$ tissue) in a favourable environment. The most favourable environment is haematoma, followed by necrotic soft-tissues and avascular cortical bone. ${ }^{10}$ Animal models of osteomyelitis invariably require some form of damage to the bone, either mechanical, chemical, or the insertion of a foreign body, to reliably establish infection. ${ }^{10,11}$ Haematogenous osteomyelitis is occasionally encountered in puppies but is very unusual in adults dogs and normally associated with systemic clinical signs of disease. ${ }^{12,13}$ The proportion of bone infections that progress to bone sequestration is unknown, but it is thought to depend on many factors including the host species, host immune status and anatomic location. ${ }^{11}$

Bone sequestration is well documented in cattle ${ }^{3,5}$ and horses ${ }^{4,14,15}$ and generally follows soft-tissue trauma to the distal limb in the absence of a fracture. The third metacarpal or metatarsal bones are most commonly affected and most lesions occur following relatively minor trauma such as lacerations, puncture wounds, or blunt trauma such as kicks. In areas of poor soft-tissue coverage such as the distal limb, minor trauma can cause periosteal disruption which may lead to avascular necrosis of the outer cortex of the bone. Simultaneous inoculation of bacteria through a contaminated wound can lead to colonization of the avascular segment of bone and sequestrum formation. Adolescent animals seem to be at higher risk, although it is not known whether this is as a consequence of differences in the thickness, vascularity and adhesion of the periosteum or these animals being more boisterous and accident prone. ${ }^{5}$ Sequestra are frequently associated with mixed infections of environmental bacteria, particularly Trueperella pyogenes (formerly known as Actinomyces pyogenes), Streptococcus and Escherichia coli. Sequestration generally appears to affect the diaphyseal regions of the long bones, involving avascular cortical fragments that are too big to be broken down or extruded through a sinus. ${ }^{10}$ Revascularization or reabsorption is unlikely to occur and surgical debridement is indicated to remove all avascular tissue and to promote resolution of the lesion. Sequestra are usually 'free-floating' and can normally be identified in situ after removal of the involucrum by their characteristic yellowish-white colour and 'pitted' appearance. ${ }^{10}$ Methylene blue flushing of sinus tracts before surgery, tetracycline label- ling or laser Doppler flowmetry may help to differentiate viable from non-viable bone at surgery. ${ }^{16,17}$

In the first case reported, it appears that soft-tissue trauma and inoculation (likely a bite from the sibling) initiated concurrent osteomyelitis in metatarsal bones two and four. Subperiosteal haematoma and exudate accumulation leading to periosteal elevation ('creeping periostitis') may have created the large avascular diaphyseal sequestra by physically disrupting the outer cortical vascular supply in addition to vascular injury induced by bacterial exotoxins and immune responses. It is possible that contaminated metatarsal fractures occurred at the time of trauma but were overlooked on the initial clinical examination, or that osteomyelitis arose from haematogenous seeding; but these were considered less likely causes. Pasteurella pneumotropica is a host-adapted to laboratory animals but can be associated with dog bites and opportunistic infections. ${ }^{18}$ Haematogenous seeding of these bacteria would likely trigger infection in multiple locations and at predictable locations in the juvenile skeleton. ${ }^{12,13}$

In the second case, an opportunistic infection appears to have occurred in a conservatively treated segmental fracture. The source of this infection is not clear from the history; it may have been an open fracture or it may have been due to haematogenous seeding of the fracture haematoma from a distant site. Considering the superficial location, the likely comminuted nature of the fracture and given the culture results, we consider most likely that the contamination came from a skin penetrating lesion at the time of fracture. The soft-tissue trauma and prolonged instability at the fracture site may have contributed to the development of chronic osteomyelitis with sequestration. $^{2,10,19}$ Colonization of an ischemic bone fragment allows persistence of the infection. Over time, reactive bone and granulation tissue forms around the dead bone fragment to isolate the septic focus. Purulent exudate eventually drains through a sinus. Case two also demonstrated the unusual process of spontaneous tibiofibular synostosis with fibula hypertrophy to bridge a segmental defect in the main weight-bearing axis of the limb.

In the third case, it is likely that a penetrating bite wound caused simultaneous sub-periosteal haematoma formation and contamination with pathogenic bacteria. Exudate accumulation is likely to have resulted in localized cortical osteonecrosis and periosteal elevation similar to that proposed to occur in large animals. Elevation of the periosteum leads to reactive new bone forming an involucrum around the sequestrum with a cloaca communicating with the abscess cavity, although in this case no soft-tissue sinus to the skin developed.

All three cases were treated by sequestrectomy, leading to complete resolution in the first and third cases and reportedly an improvement in the second case. In this second case, the sinuses resolved after surgery but mild lameness persisted in the 6 months follow-up period, presumably due to the synostosis and chronic bone deformity.

A positive culture was obtained in all three cases from the sequestrum itself (cases 1 and 3 ) or from an 
intraoperative swab of the exudate in the abscess cavity (case 2). Direct culture of the sequestrum may be more likely to yield a positive result. Thus, intraoperative sampling for bone culture is considered more sensitive and specific than a swab from a sinus tract for determining the cause of chronic osteomyelitis. ${ }^{20}$ Blood culture was not performed in our cases as at the time of presentation the osteomyelitis seemed localized and none of the patients had signs of systemic disease or pyrexia. It is possible that polymicrobic infections were present in these cases despite isolation of single bacterial species in each case; preventing sample exposure to ambient air and specific culture conditions may improve detection of obligate anaerobes. ${ }^{13,21}$

In case two, a cancellous bone graft was used to pack the abscess cavity. Cancellous bone grafting may improve revascularization of the lesion by inducing angiogenesis and contributes to healing of the bony defect created by debridement. ${ }^{22}$ Collecting an autologous cancellous bone graft prolongs surgery, increases morbidity and necessitates a second surgical approach to the donor site. Although this was considered appropriate by the surgeon at the time of treating case two, this was probably not essential as removal of the loose sequestrum was unlikely to have weakened the synostosed tibiofibular bone.

After debridement, in all three cases, the lesions were copiously flushed with $0.9 \%$ sterile saline solution alone, as this remains the golden standard for wound lavage during surgical management of osteomyelitis, despite the availability of various antiseptic agents. ${ }^{23}$

Although sequestrum removal is considered vital for resolution of chronic osteomyelitis, ${ }^{19,24}$ the duration for which antibiotic medications are required to clear residual infection is not clear. In the presented cases, the duration of antibiotic treatment was at the discretion of the surgeon, based on clinical judgement. In case one, no postoperative antibiotic medications were prescribed as we considered debridement complete and only normal, well vascularized tissue remained. The surgical site was also superficial and wound healing and lameness resolution were closely monitored in the postoperative period. In contrast, in case three, antibiotic medications were prescribed for 6 weeks until resolution was confirmed radiographically. In this case, no previous antibiotic medications had been used, the bone surrounding the lesion was considered diseased but viable and the surgical site was deep and close to a major joint. In humans, 6 weeks of antibiotic medications are generally recommended for the treatment of chronic osteomyelitis, but the optimal duration of therapy remains uncertain and treatment failures are common. ${ }^{25,26}$

\section{Conclusions}

Chronic osteomyelitis with sequestration may occur without a history of previous surgery in dogs and skin sinuses may not always be present. The prognosis appears favourable if a sequestrum can clearly be identified and be completely removed and debrided surgically.

\section{Author Contribution}

Sorrel Langley-Hobbs and Graham Hayes contributed the case material for this manuscript. All authors contributed to the drafting and revision of the manuscript.

\section{Funding}

None.

\section{Conflict of Interest}

None declared.

\section{References}

1 Johnson KA. Osteomyelitis in dogs and cats. J Am Vet Med Assoc 1994;204(12):1882-1887

2 Robinson DA. Osteomyelitis and implant-associated infections. In: Johnson S, Tobias Keds. Veterinary Surgery: Small Animal. 2nd ed. Missouri: Elsevier; 2018:775-782

3 Firth EC. Bone sequestration in horses and cattle. Aust Vet J 1987; 64(03):65-69

4 Clem MF, DeBowes RM, Yovich JV, Douglass JP, Bennett SM. Osseous sequestration in the horse. A review of 68 cases. Vet Surg 1988;17(01):2-5

5 Valentino LW, St Jean G, Anderson DE, et al. Osseous sequestration in cattle: 110 cases (1987-1997). J Am Vet Med Assoc 2000;217 (03):376-383

6 Amine B, Benbouazza K, Harzy T, Rahmouni R, Hajjaj-Hassouni N. Chronic osteomyelitis of the metacarpals. Report of a case. Joint Bone Spine 2005;72(04):322-325

7 De Smet AA, Dueland RT, Dubielzig RR. Case report 775. Canine osteosarcoma with associated avascular necrosis and sequestrum formation. Skeletal Radiol 1993;22(02):146-149

8 Ackerman N, Halliwell WH, Wingfield WE, et al. Bone infarction and sequestrum formation in a canine osteosarcoma. J Am Vet Rad Soc $1975 ; 16: 3-5$

9 Seiler G, Rossi F, Vignoli M, Cianciolo R, Scanlon T, Giger U. Computed tomographic features of skull osteomyelitis in four young dogs. Vet Radiol Ultrasound 2007;48(06):544-549

10 Braden TD. Posttraumatic osteomyelitis. Vet Clin North Am Small Anim Pract 1991;21(04):781-811

11 Tsukayama DT. Pathophysiology of posttraumatic osteomyelitis. Clin Orthop Relat Res 1999;(360):22-29

12 Cabassu J, Moissonnier P. Surgical treatment of a vertebral fracture associated with a haematogenous osteomyelitis in a dog. Vet Comp Orthop Traumatol 2007;20(03):227-230

13 Rabillard M, Souchu L, Niebauer GW, Gauthier O. Haematogenous osteomyelitis: clinical presentation and outcome in three dogs. Vet Comp Orthop Traumatol 2011;24(02):146-150

14 Booth LC, Feeney DA. Superficial osteitis and sequestrum formation as a result of skin avulsion in the horse. Vet Surg 1982; $11: 2-8$

15 Wilmink JM, van Herten J, van Weeren PR, Barneveld A. Retrospective study of primary intention healing and sequestrum formation in horses compared to ponies under clinical circumstances. Equine Vet J 2002;34(03):270-273

16 Dahners LE, Bos GD. Fluorescent tetracycline labeling as an aid to debridement of necrotic bone in the treatment of chronic osteomyelitis. J Orthop Trauma 2002;16(05):345-346

17 Duwelius PJ, Schmidt AH. Assessment of bone viability in patients with osteomyelitis: preliminary clinical experience with laser Doppler flowmetry. J Orthop Trauma 1992;6(03):327-332

18 Sahagún-Ruiz A, Granados Martinez AP, Breda LC, et al. Pasteurella pneumotropica evades the human complement system by acquisition of the complement regulators factor $\mathrm{H}$ and C4BP. PLoS One 2014;9(10):e111194 
19 Bubenik LJ. Infections of the skeletal system. Vet Clin North Am Small Anim Pract 2005;35(05):1093-1109, v

20 Akinyoola AL, Adegbehingbe OO, Aboderin AO. Therapeutic decision in chronic osteomyelitis: sinus track culture versus intraoperative bone culture. Arch Orthop Trauma Surg 2009;129(04): 449-453

21 Muir P, Johnson KA. Anaerobic bacteria isolated from osteomyelitis in dogs and cats. Vet Surg 1992;21(06):463-466

22 Pape HC, Evans A, Kobbe P. Autologous bone graft: properties and techniques. J Orthop Trauma 2010;24(Suppl 1):S36-S40
23 Tiemann AH, Hofmann GO. Wound irrigation within the surgical treatment of osteomyelitis. GMS Interdiscip Plast Reconstr Surg DGPW 2012;1:Doc08

24 Dernell WS. Treatment of severe orthopedic infections. Vet Clin North Am Small Anim Pract 1999;29(05):1261-1274, ix

25 Spellberg B, Lipsky BA. Systemic antibiotic therapy for chronic osteomyelitis in adults. Clin Infect Dis 2012;54(03):393-407

26 Conterno LO, Turchi MD. Antibiotics for treating chronic osteomyelitis in adults. Cochrane Database Syst Rev 2013;9(09): CD004439 